\title{
Use of cough swabs in a cystic fibrosis clinic
}

\author{
A C Equi, S E Pike, J Davies, A Bush
}

\begin{abstract}
We audited prospectively 322 cough swabs taken from cystic fibrosis children and compared cough swabs with concomitant sputum samples in 30 expectorating patients. A positive cough swab is a strong predictor of sputum culture. However, a negative cough swab does not rule out infection. Persistent symptoms should be further investigated.

(Arch Dis Child 2001;85:438-439)
\end{abstract}

Keywords: cystic fibrosis; cough swabs; sputum; clinic

Over the past two decades survival has improved in cystic fibrosis (CF), mainly as a result of aggressive antibiotic policies and improved nutrition. However, death is still principally caused by respiratory failure as a result of progressive suppurative lung disease. It is increasingly realised that early recognition and eradication of airway pathogens may delay the progression of lung disease. ${ }^{1}$ The majority of older patients expectorate and as sputum cultures accurately reflect lower airway secretions, antibiotic therapy can be tailored to the results of the sputum culture. ${ }^{2}$ Younger patients do not usually expectorate and so cough swabs from the upper airway are often used in an attempt to reflect lower airway secretions. Information on the value of routine cough swabs is scanty. The aim of this study was to audit prospectively the cough swabs taken from non-expectorating patients over a period of six months. In order to determine the diagnostic accuracy of cough swabs we compared cough swabs with spontaneously expectorated sputum samples in a separate group of 30 expectorating patients.

\section{Subjects and methods}

All non-expectorating patients attending a paediatric CF clinic (age range 1.3-17 years) over a six month period had a cough swab performed. The physiotherapist obtained cough swabs by asking the patient to cough onto a cotton tipped swab placed in, but not touching the posterior pharynx. Patients were divided into two groups, symptomatic and non-symptomatic. Patients were placed in the non-symptomatic group if they had either no respiratory symptoms or no increase in usual symptoms. In a different group of 30 productive adult patients, a cough swab was taken and then sputum was spontaneously expectorated. Samples were cultured for the common CF pathogens using the following media: heated blood agar, McConkey, Burkholderia Cepacia, Difco Pseudomonas, Sabaurauds Dextrose, and mannitol salt agar. Plates were incubated at $37^{\circ} \mathrm{C}$ and assessed at 24 and 48 hours. Antibiotics were commenced empirically at the clinic visit according to respiratory symptoms, but treatment was altered as directed by the result of the microbiological culture.

Cough swabs were compared to sputum samples using a Bland Altman two by two contingency table. ${ }^{3} 4$

\section{Results}

A total of 322 cough swabs were obtained from 161 patients; $87(27 \%)$ patients were symptomatic and $235(73 \%)$ were asymptomatic. Eighteen of 235 (8\%) cough swabs from asymptomatic patients were positive and isolated the following organisms: Pseudomonas aeruginosa $(\mathrm{n}=8)$, Staphylococcus aureus $(\mathrm{n}=6)$, Haemophilus influenzae $(\mathrm{n}=5)$. All these results led to a new course of antibiotics. In two patients, $P$ aeruginosa was isolated for the first time; aggressive anti-pseudomonal treatment with oral and nebulised antibiotics was commenced according to our clinic policy.

Twenty two of $87(26 \%)$ cough swabs from the symptomatic group were positive: Staphylococcus aureus $(\mathrm{n}=11)$ and $P$ aeruginosa $(n=13)$ were isolated. All these patients had empirically commenced treatment at the time of consultation. However, the cough swab results led to a change in antibiotic therapy in five patients.

In the second study, 30 patients had cough swabs compared with concomitant spontaneously expectorated sputum samples. The following organisms were isolated: $P$ aeruginosa ( $\mathrm{n}=13)$, mucoid $P$ aeruginosa $(\mathrm{n}=12)$, Staphylococcus aureus $(\mathrm{n}=7)$, Stenotrophomonas maltophilia $(\mathrm{n}=3)$, Burkholderia cepacia $(n=1)$. Twelve bacterial isolates were cultured from cough swabs; 36 isolates were cultured from the corresponding sputum sample. Eleven of $12(92 \%)$ organisms detected in the cough swabs and in the corresponding sputum sample had the same antibiotic sensitivity pattern. Cough swabs compared with sputum samples had a specificity of $100 \%$ and sensitivity of $34 \%$. The positive predictive value for a cough swab was $100 \%$ and the negative predictive value was $21 \%$.

\section{Discussion}

The principle findings of this study are firstly that a positive cough swab, although commoner in symptomatic, non-productive patients, is not uncommon in asymptomatic children with CF. Secondly, the study comparing cough swabs with spontaneously expectorated sputum implies that organisms isolated from a cough swab are representative of those found in lower airway secretions obtained from sputum culture. Therefore, a positive cough swab is strongly predictive of lower airway infection. However, a negative cough swab cannot rule out infection in the lower airway. 
The strengths of this study are in its pragmatic design and outpatient setting. The weaknesses include the lack of any bronchoalveolar lavage (BAL) data which would be considered the "gold standard" for microbiological culture of the sections. ${ }^{5}$ However, BAL is not a routine procedure and cannot be carried out in an outpatient setting; therefore we could not justify its use for this study. This study also does not address whether our practice of treatment guided by cough swabs results will affect long term outcome data in our paediatric $\mathrm{CF}$ population.

Our results conflict with a large study in which throat swabs were compared to BAL and suggested that a negative throat swab is helpful in ruling out lower airway infection. ${ }^{6}$ The investigators used oropharyngeal swabs taken under general anaesthesia; in our study we used cough swabs taken with no direct contact with the oropharynx, and all swabs were performed in an outpatient setting.
We conclude that cough swabs can provide additional useful information to direct antibiotic therapy in young, non-expectorating $\mathrm{CF}$ patients as even non-symptomatic patients may have a positive cough swab. A positive cough swab is a strong predictor of a positive sputum sample; however, a negative cough swab cannot rule out lower airway infection and persistent symptoms should be further investigated, for example by BAL.

1 Koch C, Hoiby N. Diagnosis and treatment of cystic fibrosis. Respiration 2000;67:239-47.

2 Huang NM, Van Loon EL, Shang KT. $f$ Paediatr 1961;59:512-21.

3 Altman DG, Bland JM. Diagnostic tests 2: predictive values. BMF 1994;309:102.

4 Altman DG, Bland JM. Diagnostic tests 1: sensitivity and specificity. BMF 1994;308:1552.

5 Kahn FW, Jones JM. Diagnosing bacterial respiratory infection by bronchoalveolar lavage. I Infect Dis 1987;155: 862-9.

6 Rosenfeld M, Ramsay B, et al. Diagnostic accuracy of oropharyngeal cultures in infants and young children with cystic fibrosis. Pediatr Pulmonol 1999;28:321-8.

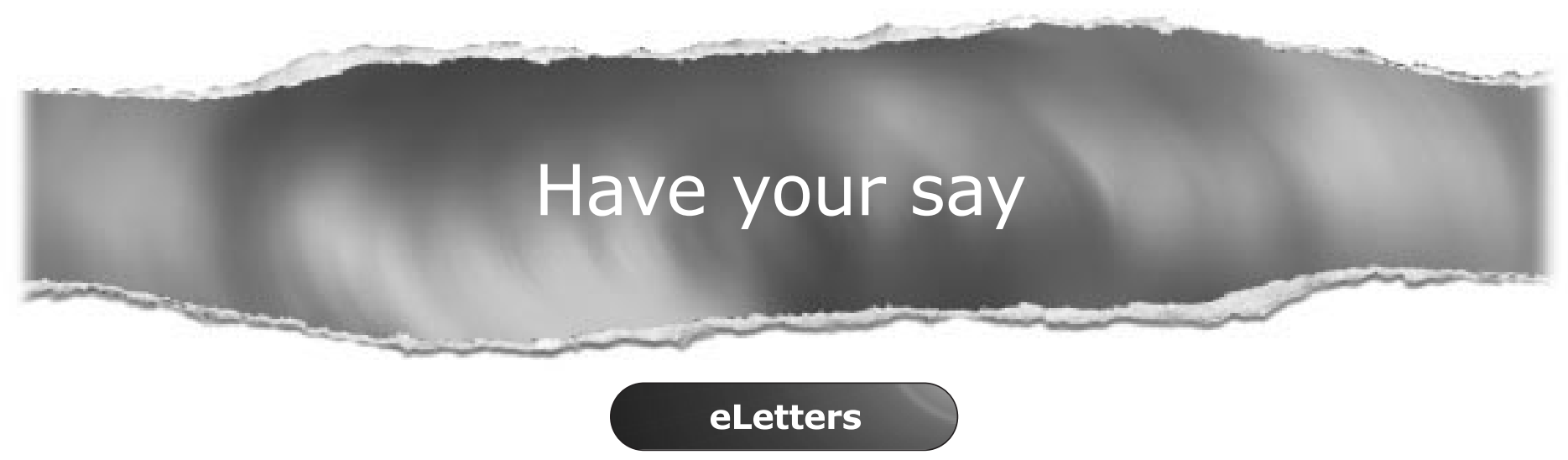

If you wish to comment on any article published in Archives of Disease in Childhood you can send an eLetter using the eLetters link at the beginning of each article. Your response will be posted on Archives of Disease in Childhood online within a few days of receipt (subject to editorial screening).

www.archdischild.com 